\title{
Language Processing in Toddlers with Permanent Congenital Sensorineural Hearing Impairment: A Preliminary Functional Magnetic Resonance Imaging (fMRI) Study
}

\author{
Prasanna Karunanayaka, ${ }^{1,2}$, Rommy Elyan ${ }^{1}$, Sara Robertson ${ }^{3}$, Marguerite Care ${ }^{4}$, John \\ Greinwald $^{5,6}$ and Scott K Holland ${ }^{2,4,5}$ \\ ${ }^{1}$ Department of Radiology (Center for NMR Research), Pennsylvania State University, USA \\ ${ }^{2}$ Pediatric Neuroimaging Research Consortium, Cincinnati Children's Hospital Research Foundation, USA \\ ${ }^{3}$ Department of Pediatrics, University of Tennessee Health Sciences Center, USA \\ ${ }^{4}$ Department of Radiology, Cincinnati Children's Hospital Medical Center, USA \\ ${ }^{5}$ Communication Sciences Research Center, Cincinnati Children's Hospital Research Foundation, USA \\ ${ }^{6}$ Division of Pediatric Otolaryngology, Children's Hospital Medical Center, University of Cincinnati College of Medicine, USA
}

*Corresponding author: Prasanna R Karunanayaka, Department of Radiology, Pennsylvania State University College of Medicine, The M. S. Hershey Medical Center, 500 University Dr., Hershey, PA, USA.

To Cite This Article: Prasanna Karunanayaka, Language Processing in Toddlers with Permanent Congenital Sensorineural Hearing Impairment: A Preliminary Functional Magnetic Resonance Imaging (fMRI) Study. 2020 - 10(1). AJBSR.MS.ID.001471. DOI: 10.34297/AJBSR.2020.10.001471.

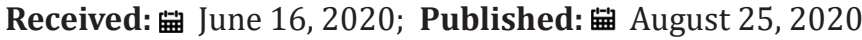

\begin{abstract}
Objective: To evaluate the utility of functional magnetic resonance imaging (fMRI) in toddlers under sedation (with permanent congenital sensorineural hearing impairment) who are candidates for Cochlear Implantation (CI).

Methods: Infants with normal hearing ( $\mathrm{NH}$ ) and with bilateral (severe to profound) sensorineural hearing impairment (HI) participated in a clinically indicated fMRI study under sedation. All subjects were 9-24 months of age. NH subjects were recruited from the clinical MRI schedule for non-hearing related scan procedures.

Results: NH infants activated language-related brain regions (superior temporal and angular gyri) under sedation. These brain regions are typically activated in older children during similar fMRI language tasks. In contrast to the NH group, the HI group exhibited strong frontal activation. This suggests different sound processing mechanisms in HI compared to the NH group.

Conclusion: Infants with residual hearing elicited greater fMRI BOLD activation in auditory and language brain regions in response to auditory stimulation. Residual hearing in infants before $\mathrm{CI}$ is known to be an effective predictor for enhanced spoken language abilities post $\mathrm{CI}$. The results of this preliminary study suggest that fMRI can be an effective clinical prescreening-tool for predicting and monitoring the benefits of CI-induced amplification-ensuring timely intervention strategies in $\mathrm{HI}$ infants.
\end{abstract}

\section{Introduction}

Undetected hearing loss in infants is known to adversely affect early speech and language acquisition-directly affecting later academic achievement, including social and emotional development in children. Early detection of sensorineural hearing

impairment (HI) in children can minimize these negative outcomes through enrollment in targeted intervention programs [1]. Universal newborn hearing and screening programs in the United States have reduced the average age of diagnosis of congenital 
hearing impairment to 3-6 months of age, increasing the likelihood for appropriate and successful early intervention in these infants [2].

Based on behavioral data, several investigations suggest that early cochlear implantation (CI) might take advantage of the inherent neuronal flexibility during critical periods of auditory based learning [3-9]. As suggested by Niparko et al., the timing of implantation must be a function of the level of hearing loss and the associated delays in spoken language ability [4]. They also reported that greater rates of improvement in language comprehension and expression are significantly correlated with both age and residual hearing at implantation. In addition, higher ratings of parent-child interactions, and higher socioeconomic status have a positive impact on the rates of improvement in language comprehension and expression measures after implantation. Behavioral studies in CI have shown notoriously high variance in result outcomes [47]. Nevertheless, the use of cochlear implants in young children has resulted in better spoken language abilities than would be predicted from pre-implantation scores [4].

Spoken language learning relies on effective hearing; developing objective tools that can evaluate residual hearing to gauge the benefits of amplification may vastly improve later spoken language capabilities in CI children. Such tools can provide timely information on when to proceed with cochlear implantation. Otoacoustic emissions (OAE), and auditory brainstem response (ABR) testing are available for objectively evaluating peripheral auditory functions from the cochlea to the lower brainstem. These two measurements can provide information about signal transduction and propagation along the central auditory nervous system pathway with excellent temporal resolution, albeit with relatively poor spatial resolution.

Functional Magnetic Resonance Imaging (fMRI) is a technique that detects brain activation using changes in the Blood Oxygenation Level Dependent (BOLD) contrast. There is a close relationship between the neuronal activity underlying brain stimulation and this BOLD contrast, which affects the MRI signal. Thus, the BOLD effect can provide indirect evidence for auditory and language stimulation in the cortex [10]. The goal of the current preliminary investigation is; therefore, to determine the feasibility and utility of fMRI in the evaluation process for $\mathrm{CI}$ in toddlers with permanent congenital sensorineural hearing impairment. This research would extend the findings of a previous study in older HI children (mean age, 49.1 months) [11].

We hypothesize that, in response to auditory stimulation, congenitally deaf infants who demonstrate higher fMRI activation in the auditory and language areas of the brain will exhibit greater improvements in post-implant hearing threshold tests. CI infants who demonstrate lower activation in those brain areas are expected to under-perform on the same tests.

\section{Methods}

\section{Subjects}

Forty-three toddlers, aged 9 24 months, participated in a clinically indicated MRI brain study under sedation [either with Propofol $(n=21)$, Nembutal $(n=17)$ or Sevoflurane $(n=5)]: 22$ normal hearing $(\mathrm{NH})$ and 21 with bilateral severe to profound sensorineural hearing impairment (HI). NH subjects were recruited from the clinical MRI schedule and met the inclusion criteria: gestational age of at least 36 weeks and normal otoacoustic hearing emissions. A neuroradiologist reviewed the anatomical brain scans to confirm that the neuroanatomy of the auditory cortex appeared within normal limits for the infants' age (Tables $1 \mathrm{a} \& 1 \mathrm{~b}$ ).

Table 1a: Table of Subjects.

\begin{tabular}{|c|}
\hline Total \# of subjects with normal hearing $(\mathrm{NH})=22$ \\
\hline Total \# of subjects with hearing impairment $(\mathrm{HI})=21$ \\
\hline $\mathrm{HI}(<24$ months old $)$ and with pre- and post- hearing data $=11$ \\
\hline $\mathrm{HI}(<24$ months old $)$ and sedated with Propofol and with pre- and post- \\
hearing data $=8$
\end{tabular}

Table 1b: MRI referral reasons for normal hearing subjects.

\begin{tabular}{|c|c|}
\hline Number of Subjects & Referral \\
\hline 8 & Seizures \\
\hline 5 & Visual/Eye Pathologies \\
\hline 2 & R/O Mass \\
\hline 2 & Pierre Robin Sequence \\
\hline 1 & Torticollis \\
\hline 1 & Vomiting/Feeding Disorder \\
\hline 1 & Obstructive Sleep Apnea \\
\hline 1 & Lower Extremity Clonus \\
\hline 1 & \\
\hline
\end{tabular}

*Note: Average age for normal hearing subjects were $12.25 \pm 2.88$ (range 7 17) months.

The study had the Cincinnati Children's Hospital Medical Center (CCHMC) Institutional Review Board's approval, and the written and informed consent of the guardians of all subjects. The fMRI scanning was added to the end of clinical scans.

\section{fMRI Paradigms and Image Acquisition}

Hemodynamics Unrelated to Sounds from Hardware (HUSH) is an event-related fMRI acquisition and post-processing technique that allows auditory stimuli to be presented during completely silent gradient intervals [12,13]. The stimulation paradigm consisted of narrow band noise (NBN), speech, or silence ( 5 second segments). They were all interleaved with 6 second acquisition periods [12]. All images were acquired on a 3.0 Tesla Siemens Trio MRI scanner with the following parameters: TR/TE $=2000 / 38$ msec., flip angle $=77.60,6$ sec. acquisition period, 5 sec. stimulus duration, 5 sec. control period. Each stimulus acquisition epoch lasted for 11 seconds (Figure 1). 


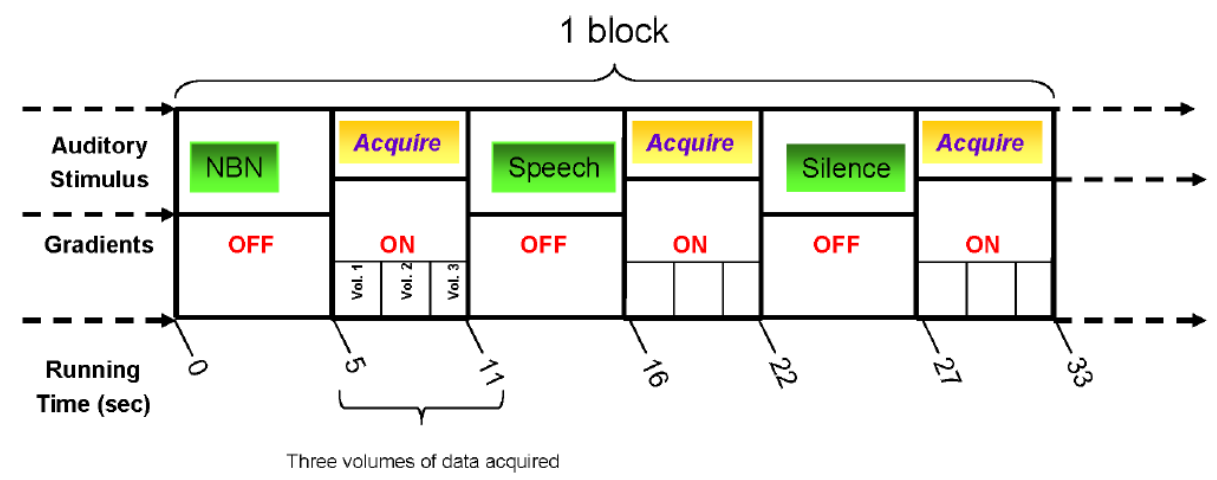

Figure 1: The three-phase event related fMRI auditory paradigm in which the stimuli are presented during completely silent gradient intervals. During each phase, 3 Echo Planar Imaging (EPI) volumes were acquired at 2 seconds per volume; each volume comprising 25 slices of $4 \mathrm{~mm}$ thickness and $3 \times 3 \mathrm{~mm}$ in-plane resolution. The total fMRI acquisition time was 11 minutes (NBN: Narrow Band Noise) [12].

\section{Sedation}

The CCHMC routinely sedates pediatric patients under the age of 7 years for clinical MRI procedures. The infants were sedated for the clinical neuroimaging exam using either Propofol (IV drip 200-300 $\mathrm{mcg} / \mathrm{Kg} / \mathrm{min}$, with $8 \%$ Sevoflurane induction for most subjects), or Nembutal (3.0-7.0 mg/kg oral or IV) as determined by the anesthesiologist participating in the study.

\section{Audiometric Measurements}

Audiometric data, including behavioral and speech awareness thresholds (SAT), were obtained from each infant prior to cochlear implantation. The Minimal Response Level Average (MRLA) was calculated by averaging the available threshold values at $0.5,1,2$, and $4 \mathrm{kHz}$, respectively. If a child did not respond to the maximum sound level of the audiometer, the maximum was taken as the reading-although the true threshold was likely higher. Behavioral thresholds were also collected post implantation. For bilateral CI recipients, the calculation of MRLA was based on thresholds obtained with both CIs. For unilateral recipients, the average was calculated based on thresholds obtained with either their left or right CIs.

The difference in pre- and post-MRLA was used in a subsequent correlation analysis with brain activation. In the case where preMRLA was not available, the pre- pure tone average (PTA) in the sound-field was used. If the subject had received bilateral (sequential or simultaneous) implants, the pre-sound-field PTA was used. Alternatively, the left (or right) MRLA average was used in cases where the sound-field was not available. Because the correlation was performed on a small subset of infants (less than 24 months of age) sedated with Propofol ( $n=8)$, a non-parametric Spearman's rank correlation method was used to examine the relationship between auditory activation and improvement in MRLA after CI.

\section{Data Processing}

Data processing and statistical analyses were performed using the CCHMC's Image Processing Software (CCHIPS) [14]. The EPI images were corrected for Nyquist ghosting and geometrical distortion [14]. Signal drift was corrected using a quadratic baseline correction [14]. Motion artifacts were corrected using a pyramid iterative co-registration algorithm [14]. All infant brains were normalized to the CCHMC's infant brain template [15]. The preprocessing and normalization procedures were performed based on algorithms specifically developed for this infant cohort [14].

\section{Statistical Analysis}

The General Linear Model was used to construct individual Z-score maps for each contrast [16]. Consistent with other studies, we observed both positive and negative activation in central auditory regions under the influence of sedation during fMRI $[11,17]$. A voxel-wise random effects analysis was performed to find regions of significant activation for these groups, separately [14]. Composite group activation maps were spatially filtered using a Gaussian kernel of $4 \mathrm{~mm}$. Based on an ROI containing the language/frontal brain regions, a Monte-Carlo simulation was used to determine the corrected $p$-values for these maps [14].

\section{ROI Analysis}

The first ROI was defined manually [18] by a pediatric neuroradiologist. It encompassed the entirety of the primary auditory cortex (A1), located within the transverse temporal gyrus (Heschl's gyrus and Brodmann Area 41) on the group anatomical image, for the normal hearing group. The second ROI was defined based on the functional map for the story vs. silence contrast in the NH group, and encompassed higher-order language processing brain regions-in addition to the primary auditory cortex defined in the first ROI. 


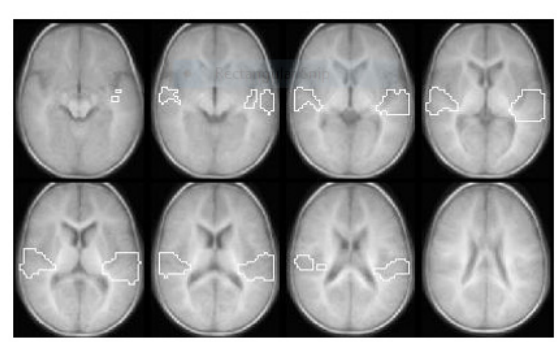

Figure 2: The functional ROI (outlined in white) overlaid on an anatomic image combining the functionally defined ROI based on the story vs. silence contrast with the manually defined primary auditory cortex region.

Figure 2 shows the ROI used in the current analysis. This ROI was generated by combining the first and the second ROIs described earlier. The fMRI activation was parameterized by counting the number of pixels within the functionally defined ROI that exceeded a pre-defined threshold $(\mathrm{p}<0.05)$ for each subject. Within the ROI, the total number of supra-threshold pixels (both positive and negative) were tallied and used in the linear regression of pixel count vs. change in hearing threshold, as measured by MRLA. The pixel counting approach is methodologically comparable to standard lateralization index (LI) calculations in functional neuroimaging studies [19-21]. Out of the 21 CI subjects enrolled in the study, only eleven subjects had a 24-month post-implant hearing threshold measurement at the time of writing this report. Data from these participants was included in a group analysis and used to construct composite activation maps for the HI cohort. In order to minimize variability due to the different sedatives, doses, and timing: only those participants sedated with Propofol (8 out of 11), who were less than 24 months of age, were included in the prevs. post-regression analyses.

\section{Results}

Figure 3 shows the statistical parametric map (composite Z-score map) for group activation (corrected $p<0.05$ ) for story processing vs. silence contrast across the entire normal hearing (NH) group $(\mathrm{n}=22)$. Similar to our previous reports on story processing in older and awake children, the superior temporal gyrus (STG) is activated in the NH group [22]. As tabulated in Table 2 , the group analysis [with the generalized linear model (GLM)] also detected activation in the visual cortex for sedated infants with normal hearing $(\mathrm{NH})$. (a)

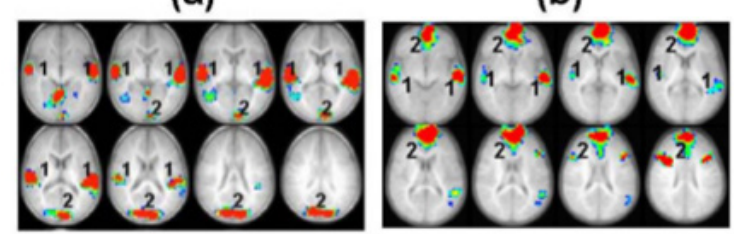

(c)

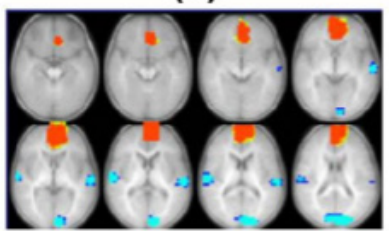

Figure 3: Composite Z-score maps (in axial plane) showing significantly activated brain regions $(p<0.05)$ during language processing (across the slice range 2.5 to $+37.5 \mathrm{~mm}$ ) for the story vs. silence contrast in (a) the normal hearing $(\mathrm{NH})$ control group $(n=22)$ and $(b)$ the hearing impaired $(\mathrm{HI})$ group [less than 24 months of age ( $\mathrm{n}=11)$ ]. (c) Group difference map for the story vs. silence contrast across the $\mathrm{NH}$ and $\mathrm{HI}$ subjects $(\mathrm{p}<0.05)$. Red indicates areas where activation in the $\mathrm{HI}$ group is greater compared to the $\mathrm{NH}$ group. Blue indicates areas where the $\mathrm{NH}$ group show greater activation compared to the $\mathrm{NI}$ group. Tables 2 and 3 include activated brain regions in figures $(a)$ and $(b)$.

This region of activation was not previously found in awake children performing a similar task [22]. The other contrast we were able to examine, based on data from the 3-phase fMRI paradigm, was between narrow band noise (NBN) and silence. The NBN vs. silence contrast in the infant groups examined did not exhibit consistent positive activation across cohorts; thus, we have not included this contrast in any further analyses. 
Table 2: Activated brain regions shown in Figure 3a

\begin{tabular}{|c|c|c|c|}
\hline Number & Brodmann's Area & Brain Region & $\mathbf{X , ~}, \mathbf{Z}$ \\
\hline \multirow{2}{*}{1} & $21,22,41$ & $\begin{array}{c}\text { L. middle temporal gyrus and superior temporal gyrus, trans- } \\
\text { verse temporal area }\end{array}$ & $62,-14,8$ \\
\cline { 2 - 4 } & & $\begin{array}{c}\text { R. middle temporal gyrus, superior temporal gyrus, transverse } \\
\text { temporal area }\end{array}$ & $-58,-22,13$ \\
\hline 2 & 18,19 & L/R. Occipital lobule & $-18,-94,33$ \\
\hline
\end{tabular}

Table 3: Activated brain regions shown in Figure 3b.

\begin{tabular}{|c|c|c|c|}
\hline Number & Brodmann's Area & Brain Region & $\mathbf{X , Y , Z}$ \\
\hline \multirow{2}{*}{1} & \multirow{3}{*}{$21,22,41$} & $\begin{array}{c}\text { L. middle temporal gyrus and superior temporal gyrus, } \\
\text { transverse temporal area }\end{array}$ & $62,-18,-3$ \\
\cline { 2 - 4 } & & $\begin{array}{c}\text { R. middle temporal gyrus and superior temporal gyrus, } \\
\text { transverse temporal area }\end{array}$ & $-74,-10,-3$ \\
\hline 2 & 10 & L/R. Frontal lobule & $-2,62,38$ \\
\hline
\end{tabular}

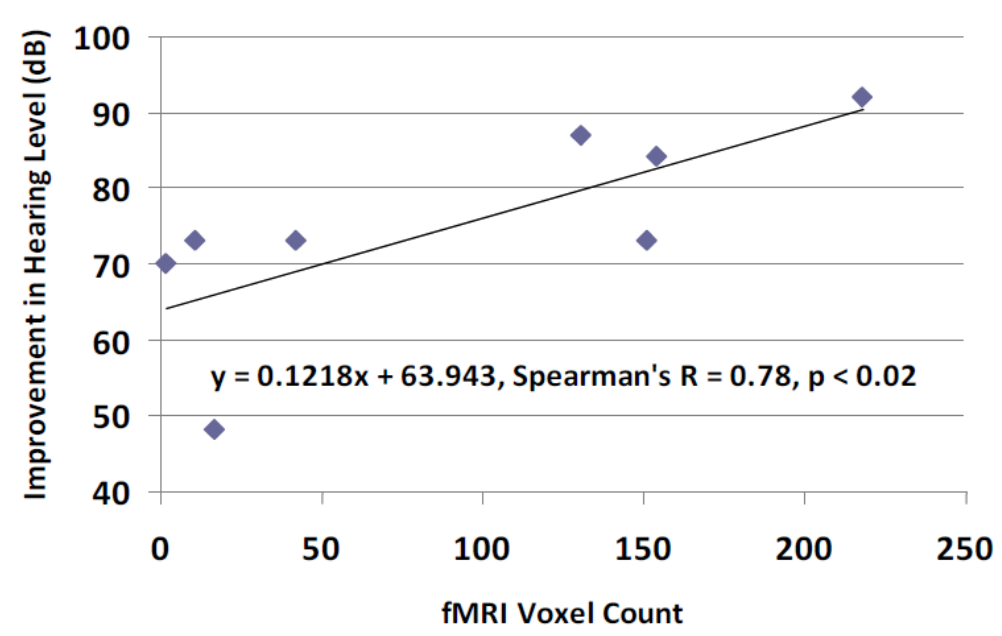

Figure 4: Improvement in hearing level vs. pre-implant fMRI. Hearing levels (MRLA, dB) as measured before and after cochlear implantation vs. activation in the ROI shown in Figure 1 for the group of $\mathrm{HI}$ infants $<24$ months of age at time of scanning. These $\mathrm{HI}$ subjects $(n=8$, mean age at scanning $=13.75 \mathrm{mo}$.) were sedated with Propofol $(200 \mathrm{mg} / \mathrm{Kg} / \mathrm{min})$. The Spearman's correlation coefficient between $\mathrm{fMRI}$ and $\mathrm{HL}$ is $r=0.78(p$ $<0.02)$.

We used the stories vs. silence contrast in the HI group (n=11) to construct the composite Z-score map shown in Figure 3b. Although HI infants demonstrated lower BOLD activation in auditory and language areas (in response to speech stimuli) than the NH group, consistent bilateral activation is seen in the middle and superior temporal gyri (BA21 \& 22), the transverse temporal gyrus (BA41), and Heshl's Gyrus (Figures 3a \& 3b). The activated brain regions in the HI group are tabulated in Table 3, including Brodmann's Area designations, anatomical regions, and normalized coordinates in the CCHMC 76 framework [15].

To better visualize differences between the NH and HI groups, we computed the statistical difference map between groups for the stories vs. silence contrast (Figure 3c). Red areas indicate regions where the HI group had significantly higher activation $(\mathrm{p}<0.05)$ compared to the NH group. Similarly, areas where the activation in the $\mathrm{NH}$ group is higher than the HI group are shown in blue $(\mathrm{p}<$ 0.05).
Using HI subjects ( $<24$ months old) that were sedated with Propofol (Table 1a), we analyzed the brain activity using the following method. We counted the number of pixels that exceeded the $\mathrm{p}<0.05$ threshold within the functionally defined ROI shown in Figure 2. This count included both the positive and negative pixels that exceeded the $\mathrm{p}<0.05$ threshold. This ROI spanned 3 to $5 \mathrm{fMRI}$ slices (nominal slice thickness: $5 \mathrm{~mm}$ ). Figure 4 shows the linear regression between this pixel count and the change in the average hearing threshold $(\mathrm{p}<0.02)$. Note: At the time of fMRI scanning, the correlation between the SAT and MRLA scores was $r=0.83(p<$ 0.02) in these $\mathrm{HI}$ infants.

\section{Discussion}

The use of fMRI to investigate brain activation in infants and toddlers is relatively new. Difficulties associated with conducting infant fMRI studies have contributed to this dearth [23]. Nevertheless, investigations of the infant brain using noninvasive 
techniques such as electroencephalography (EEG), magnetoencephalography (MEG) and near-infrared spectroscopy (NIRS) are advancing rapidly $[23,24]$. While there has been documented success scanning young children with behavioral interventions alone [25], for purposes of clinical efficiency, the most common method of minimizing movement of children between 5 months to 7 years old during scanning involves the use of sedation [26,27].

A study by Martin et al. [17] concluded that the immature vascular system in neonates is the leading factor in determining the polarity of the BOLD signal, and that local cerebral blood flow (CBF) may be the dominant factor in determining the strength of the BOLD signal. Differences in BOLD effects are exacerbated when sedatives are taken into account [28]; therefore, deciphering BOLD signal behavior in infant brains presents a challenging problem that may very well determine the future clinical applicability of fMRI in infants [29].

The BOLD signal should exhibit a partial linear relationship with the concentration of anesthetic agent-at least in the case of pentobarbital [27]. This does not; however, apply to all anesthetics, as some have exhibited non-linear relationships [30,31]. Therefore, BOLD signals should be investigated in a sedative-dependent manner: taking linear and non-linear effects into account. In order to minimize the variability of the BOLD signal due to the effects of different sedatives in our study, we limited the pre- vs. postregression analyses to only those infants sedated with Propofol.

We compared activation in $\mathrm{NH}$ infants from the current study to those found in older, awake children performing the same three-phase, event-related, HUSH fMRI auditory paradigm. This comparison could provide some insight into the cortical processing of auditory inputs in sedated toddlers. As shown in Figure 5, more extensive activation is found in the awake, older children, including areas in the dorsolateral prefrontal cortex and the anterior/ posterior cingulate cortex. It has been shown that the anterior cingulate cortex (ACC), and neighboring areas in the frontal medial wall, play a role in monitoring and controlling goal-directed behavior [32]. However, both cohorts show bilateral activation in the primary auditory cortex and planum temporale. The more extensive activation seen in awake children is likely to be associated with memory, monitoring, and attention resources engaged with the active response to the stimulus [32,33]. We hypothesize that the activation pattern seen in sedated $\mathrm{NH}$ infants is, in fact, languagerelated and not an artifact attributed to sedation.

A relatively higher BOLD activation was detected in the story vs. silence contrast compared to the NBN vs. silence contrast (not shown). These findings are consistent with a speech perception study in sleeping infants where significantly higher BOLD activation was detected in language-related areas during forward speech than backward speech [26]. Our findings provide concrete evidence that the infant brain responds differentially to speech and non-speech sounds even under sedation. The differential effect may be enhanced in sedated infants due to the depressing effects of sedation on auditory BOLD signals [34]. We hypothesize that, when considering the immature vascular systems of infants and manipulating the sedative doses, fMRI could be an effective clinical tool in this very young population: a hypothesis that is partially supported by a prior study [30].

It has been shown that auditory information is still being processed in the STG, even at Propofol plasma concentrations of $2 \mathrm{mg} / \mathrm{ml} / \mathrm{min}$ [35]. These surviving areas include Heschl's gyrus, and adjoining brain regions (BA 41, 42, 22) primarily involved in very basic auditory information processing mechanisms-including the regions found in this study (Figures 3a \& 3b). In contrast, it has been reported that areas of higher-order auditory information processing may lose activation (e.g., music-induced) with increasing doses of Propofol [36]. Of note, in the current study, Propofol did not obliterate the BOLD signal in the primary auditory cortex in response to acoustic stimulation. However, this region showed far more activation for the story processing vs. silence contrast. This suggest that infants may process auditory information of speech more effectively under sedation.

The activation patterns observed in this cohort of children, with severe to profound hearing impairments (Figure $3 \mathrm{~b}$ ), appear to replicate the typical activation patterns found in the auditory/ language cortex observed in normal hearing children under sedation (Figure 3a) and in awake, older children with normal hearing (Figure 5) [33]. The HI group shows clear frontal activation not observed in the $\mathrm{NH}$ group. A possible explanation for the increased frontal activation in the HI group could be related to the response of these children to the novel auditory stimulus. Because of the high intensity sound levels used for auditory stimulation in the HI children ( $~ 10 \mathrm{~dB}$ above the measured hearing threshold), the children may be receiving a rare auditory stimulus that generates an attention response with concomitant activation in anterior frontal regions in close proximity to the anterior cingulate gyrus: a region known to be associated with attention and attention deficits.

Similar to other studies [17,37], we also observed that central auditory activation can be both positive and negative during fMRI, when under sedation. However, several explanations have been presented regarding the common occurrence of a negative BOLD effect in infants and toddlers [38]. It is postulated that negative activation in infants corresponds to a decrease in blood oxygenation preceding increased cortical activity in a localized group of neurons $[39,40]$. To be specific, increased neuronal metabolism is not accompanied by a concomitant increase in blood flow to deliver 
the additional oxygen required to support the increased cerebral metabolic rate. The observed response pattern in this study can either be attributed to this age-related neurobiological effect, or to the use of sedation. In the current analysis, this issue has been partially addressed by considering all voxels that demonstrate a significant change in blood oxygenation (both positive and negative) from the baseline (or resting state) as having some involvement in response to the auditory stimulus.

Similar rates of positive and negative activation in auditory brain regions were found in the $\mathrm{NH}$ group, regardless of the form of sedation. Therefore, it is reasonable to assume that the incidence of negative activation is not due to sedation or hearing impairment in this study. We hypothesize that the mixture of positive and negative activation in this cohort is a consequence of the immature cerebrovascular system in subjects aged between 1 and 2 years. Compared to other measures of activation (e.g. peak activation or mean activation), the number of active voxel counts is more suitable for characterizing the fMRI responses observed in the current investigation.

Finally, in addition to the study mentioned earlier, a comparable pattern of auditory/language activation has been observed in a large cohort $(n=323)$ of awake NH children and adolescents (ages between 5 to 18 years) performing a very similar story processing task [41]. Although the activation in the sedated NH infants in this report seems to be attenuated and to contain a mixture of positive and negative activation, the similarity of the distributions of activated voxels in the functionally defined ROI provides reassurance that the observed patterns correspond to the story stimulus administered to these HI infants.

\section{Limitations}

A limitation of this study is the use of different sedatives during fMRI scanning. This may be a contributing factor to the observed variability in fMRI activation-affecting the correlation analysis between the fMRI activation (in the A1 ROI) and the postimplantation hearing thresholds. Additionally, significant neural development takes place in the brain during the age range of subjects included in this study, adding another dimension of variability to the results [42]. However, the correlation between activation in the auditory ROI and improvement in hearing thresholds $(r=0.78)$ for toddlers (under 24 months of age and sedated with Propofol) is convincing (see Figure 4 for details). In fact, this correlation seems to transcend the influences of sedation, duration of deafness, and other confounding factors, at least in the case of Propofol.

Regardless of the sedatives used, doubts have been raised about assuming an invariant Hemodynamic Response Function (HRF) in awake subjects during fMRI [43]. Therefore, methods that do not assume a fixed HRF a priori (e.g. Independent Component Analysis
[ICA]) may be more suitable to tease out brain activation patterns under sedation [41]. One promising alternative to fMRI would be the Near-infrared spectroscopy (NIRS) with diffuse optical tomography (DOT) approaches, where any effects of sedation could be removed from subsequent analyses altogether [24,44-46].

It is known that language measures after cochlear implantation can exhibit notoriously high variance [4-6]. The current preliminary report avoided this issue. Future research in our lab will investigate whether pre-implant fMRI activation can be used to predict language outcome measures in $\mathrm{HI}$ infants following cochlear implantation [4].

\section{Conclusion}

This preliminary report demonstrates the feasibility of fMRI for the assessment of auditory cortical function in severe to profound $\mathrm{HI}$ infants under sedation. The pre- and post-correlation analysis provides convincing evidence: $\mathrm{HI}$ infants, who demonstrate greater fMRI activation in auditory/language regions in response to auditory stimulation will exhibit the greatest improvement in posthearing thresholds. Since residual hearing before implantation is an effective predictor of the rate increase in the acquisition of spoken language after cochlear implantation, fMRI can be used as an objective tool to monitor the benefits of amplification, and as a guide for timely clinical intervention in HI children. Future research should further investigate the full capacity of fMRI as an objective diagnostic tool that is safe and effective for predicting CI outcomes in prelingual $\mathrm{HI}$ infants.

\section{Acknowledgments}

The study was supported by a grant from the U.S. National Institute of Deafness and Communication Disorders, R01-DC07186 (P.I. SKH), Pennsylvania Department of Health using Tobacco CURE Funds and the Department of Radiology, Penn State College of Medicine.

\section{References}

1. Connor CM, Craig HK, Raudenbush SW, Heavner K, Zwolan TA (2006) The age at which young deaf children receive cochlear implants and their vocabulary and speech-production growth: is there an added value for early implantation? Ear and Hearing 27(6): 628-644.

2. Nelson HD, Bougatsos C, and Nygren P (2008) Universal newborn hearing screening: systematic review to update the 2001 US Preventive Services Task Force Recommendation. Pediatrics 122(1): 266-276.

3. Waltzman SB, Roland JT (2005) Cochlear implantation in children younger than 12 months. Pediatrics 116(4): 487-493.

4. Niparko JK, Emily AT, Thal DJ, Laurie SE, Wang NY, et al. (2010) Spoken language development in children following cochlear implantation. JAMA 303(15): 1498-1506.

5. Dettman SJ, Pinder D, Briggs RJS, Dowell RC, Leigh JR (2007) Communication development in children who receive the cochlear implant younger than 12 months: risks versus benefits. Ear Hear 28(2 Suppl): 11S-18S. 
6. Geers A, Emily T, Moog J, Chris B (2008) Long-term outcomes of cochlear implantation in the preschool years: from elementary grades to high school. Int J Audiol 47(Suppl 2): S21-S30.

7. Holt RF, Svirsky MA (2008) An exploratory look at pediatric cochlear implantation: is earliest always best? Ear Hear 29(4): 492-511.

8. Nicholas JG, Geers AE (2007) Will they catch up? The role of age at cochlear implantation in the spoken language development of children with severe to profound hearing loss. J Speech Lang Hear Res 50(4): 1048-1062.

9. Tobey EA, Geers AE, Chris B, Altuna D, Gabbert G (2003) Factors associated with development of speech production skills in children implanted by age five. Ear Hear 24(1 Suppl): 36S-45S.

10. Huettel SA, Song AW, McCarthy G (2004) Functional Magnetic Resonance Imaging. Sunderland, MA.: Sinaur Assoc. Inc.

11. Patel AM, Lisa DC, Jennifer R, Vincent S, Choo D, et al. (2007) Functional magnetic resonance imaging of hearing-impaired children under sedation before cochlear implantation. Arch Otolaryngol Head Neck Surg 133(7): 677-683.

12. Schmithorst VJ, Holland SK (2004) Event-related fMRI technique for auditory processing with hemodynamics unrelated to acoustic gradient noise. Magn Reson Med 51(2): 399-402.

13. Eden GF, Joseph JE, Brown HE, Brown CP, Zeffiro TA (1999) Utilizing hemodynamic delay and dispersion to detect fMRI signal change without auditory interference: the behavior interleaved gradients technique. Magn Reson Med 41(1): 13-20.

14. Schmithorst V, Holland SK, Dardzinski BJ (2010) CCHIPS: Cincinnat Children's Hospital Imaging Processing Software.

15. Altaye M, Holland SK, Wilke M, Gaser C (2008) Infant brain probability templates for MRI segmentation and normalization. Neuroimage 43(4): 721-730.

16. Friston KJ, Holmes AP, Worsley KJ (1999) How many subjects constitute a study? Neuroimage 10(1): 1-5.

17. Martin E, Joeri P, Thomas L, Dimitrios E, Deborah V (1999) Visual Processing in Infants and Children Using Functional Magnetic Resonance Imaging. Pediatric Research 46: 135-140.

18. Penhune VB, Cismaru R, Dorsaint PR, Petitto LA, Zatorre RJ (2003) The morphometry of auditory cortex in the congenitally deaf measured using MRI. Neuroimage 20(2): 1215-1225.

19. Binder JR, Stephen MR, Thomas AH, Frost JA, Peter AB, et al. (1995) Lateralized human brain language systems demonstrated by task subtraction functional magnetic resonance imaging. Arch Neurol 52(6): 593-601.

20. Holland SK, Plante E, Byars AW, Strawsburg RH, Schmithorst VJ, et al. (2001) Normal fMRI brain activation patterns in children performing a verb generation task. Neuroimage 14(4): 837-843.

21. Szaflarski JP, Holland SK, Schmithorst VJ, Byars AW (2006) fMRI study of language lateralization in children and adults. Hum Brain Mapp 27(3): 202-212.

22. Karunanayaka PR, Holland SK, Schmithorst VJ, Ana Solodkin, Chen EE, et al. (2007) Age-related connectivity changes in fMRI data from children listening to stories. Neuroimage 34(1): 349-360.

23. Kuhl P, Maritza RG (2008) Neural substrates of language acquisition. Annu Rev Neurosci 31: 511-534.

24. Hull R, Bortfeld H, Koons S (2009) Near-Infrared Spectroscopy and Cortical Responses to Speech Production. Open Neuroimag J 3: 26-30.

25. Vannest J, Akila R, Cicchino ND, Julie FH, Simpson SM et al. (2014) Factors determining success of awake and asleep magnetic resonance imaging scans in nonsedated children. Neuropediatrics 45(6): 370-377.

26. Ghislaine DL, Stanislas D, Lucie HP (2002) Functional neuroimaging of speech perception in infants. Science 298(5600): 2013-2015.

27. Martin E, Thiel T, Joeri P, Thomas L, Dimitri E, et al. (2000) Effect of Pentobarbital on Visual Processing in Man. Human Brain Mapping 10(3): 132-139.

28. Di Francesco MW, Sara AR, Karunanayaka P, Holland SK (2013) BOLD fMRI in infants under sedation: comparing the impact of pentobarbital and propofol on auditory and language activation. Journal of Magnetic Resonance Imaging 38(5): 1184-1195.

29. Wilcox T, Bortfeld H, Rebecca W, Eric W, Jennifer A, et al. (2009) Hemodynamic changes in the infant cortex during the processing of featural and spatiotemporal information. Neuropsychologia 47(3): 657662.

30. Marcar VL, Schwarz U, Martin E, Loenneker T (2006) How depth of anesthesia influences the blood oxygenation level-dependent signal from the visual cortex of children. AJNR 27(4): 799-805.

31. Marcar VL, Loenneker T (2004) The BOLD response: a new look at an old riddle. Neuroreport 15(13): 1997-2000.

32. Brown JW, Braver TS (2005) Learned predictions of error likelihood in the anterior cingulate cortex. Science 307(5712): 1118-1121.

33. Vannest JJ, Karunanayaka PR, Altaye M, Schmithorst VJ, Plante EM, et al. (2009) Comparison of fMRI data from passive listening and activeresponse story processing tasks in children. J Magn Reson Imaging 29(4): 971-976

34. Dueck MH, Aloys O, Wedekind C, Paul M, Ulf Boerner (2003) Propofol impairs the central but not the peripheral part of the motor system. Anesth Analg 96(2): 449-455.

35. Engelhard K, Werner C, Mollenberg O, Kochs E (2001) S(+)-ketamine/ propofol maintain dynamic cerebrovascular autoregulation in humans. Can J Anaesth 48(10): 1034-1039.

36. Dueck MH, Petzke F, Gerbershagen HJ, Paul M, Hesselmann V, et al. (2005) Propofol attenuates responses of the auditory cortex to acoustic stimulation in a dose-dependent manner: a FMRI study. Acta Anaesthesiol Scand 49(6): 784-791.

37. Altman NR, Bernal B (2001) Brain activation in sedated children: auditory and visual functional MR imaging. Radiology 221(1): 56-63.

38. Martin E, Marcar VL (2001) Functional MR imaging in pediatrics. Magn Reson Imaging Clin N Am 9(1): 231-246.

39. Frahm J, Gunnar K, Merboldt KD, Andreas K (1996) Dynamic uncoupling and recoupling of perfusion and oxidative metabolism during focal brain activation in man. Magn Reson Med 35(2): 143-148.

40. Magistretti PJ, Pellerin L (1999) Cellular mechanisms of brain energy metabolism and their relevance to functional brain imaging. Philos Trans R Soc Lond B Biol Sci 354(1387): 1155-1163.

41. Schmithorst VJ, Holland SK, Plante E (2006) Cognitive modules utilized for narrative comprehension in children: a functional magnetic resonance imaging study. Neuroimage 29(1): 254-266.

42. Kuhl PK (2004) Early language acquisition: cracking the speech code. Nat Rev Neurosci 5(11): 831-843.

43. Handwerker DA, Ollinger JM, Desposito M (2004) Variation of BOLD hemodynamic responses across subjects and brain regions and their effects on statistical analyses. Neuroimage 21(4): 1639-1651.

44. Bortfeld H, Wruck E, Boas DA (2007) Assessing infants' cortical response to speech using near-infrared spectroscopy. Neuroimage 34(1): 407415. 
45. Taga G, Homae F, Watanabe H (2007) Effects of source-detector distance of near infrared spectroscopy on the measurement of the cortical hemodynamic response in infants. Neuroimage 38(3): 452-460.
46. Alexander BGS, Bortfeld H, Huppert TJ, Beauchamp MS, Tonini RE, et al. (2010) Neuroimaging with near-infrared spectroscopy demonstrates speech-evoked activity in the auditory cortex of deaf children following cochlear implantation. Hear Res 270(1-2): 39-47. 